\title{
Multilinguales
}

\section{Sémiotique narrative de la joie dans The River between de Ngugi Wa Thiong'o}

Semiotic Narrative of Joy in Ngugi Wa Thiong'o's The River Between

\section{Abib Sene}

\section{(e) OpenEdition}

\section{Journals}

\section{Édition électronique}

URL : http://journals.openedition.org/multilinguales/1322

DOI : 10.4000/multilinguales. 1322

ISSN : 2335-1853

\section{Éditeur}

Université Abderrahmane Mira - Bejaia

\section{Édition imprimée}

Date de publication : 1 décembre 2014

Pagination : 134-169

ISSN : 2335-1535

\section{Référence électronique}

Abib Sene, "Sémiotique narrative de la joie dans The River between de Ngugi Wa Thiong'o »,

Multilinguales [En ligne], 4 | 2014, mis en ligne le 01 décembre 2014, consulté le 17 septembre 2019.

URL : http://journals.openedition.org/multilinguales/1322 ; DOI : 10.4000/multilinguales.1322

Ce document a été généré automatiquement le 17 septembre 2019.

\section{cc)}

Multilinguales est mise à disposition selon les termes de la Licence Creative Commons Attribution Pas d'Utilisation Commerciale - Pas de Modification 4.0 International 


\title{
Sémiotique narrative de la joie dans The River between de Ngugi Wa Thiong'o
}

\author{
Semiotic Narrative of Joy in Ngugi Wa Thiong'o's The River Between
}

Abib Sene

L'excision et la circoncision sont des rituels traditionnels, qui consistent en l'ablation d'une partie de l'appareil génital, pratiqués dans des ethnies africaines qui les vivent comme une purification (dioulnouder). Il en est ainsi des Halpulars au Sénégal jusqu'aux Kikuyu au Kenya en passant par les Bambara du Mali. De telles pratiques, notamment l'excision, a fait l'objet de réflexion contradictoire chez beaucoup d'universitaires et d'écrivains comme Awa Thiam (1978), Nuruddin Farah (1981) et Ngugi Wa Thiong'o $(1965,1989)$.

Nous nous intéressons à la position de Ngugi Wa Thiong'o telle qu'elle se dégage de son ouvrage The River Between (désormais RB) dans lequel l'accomplissement des deux rituels de la croyance traditionnelle, l'excision et la circoncision, à travers le parcours de deux personnages (Muthoni et Waiyaki), est confronté à un deni de la religion chrétienne dans la société Kikuyu au kenya.

Le dessein de se faire exciser envahit et obnubile la fille du pasteur Joshua. Son père, un révérend, ne pourra jamais cautionner sa décision de se faire exciser : "but father will not allow it» $(\mathrm{RB}, 25)^{1}$. Du nuage de cette rupture, se dégage l'expression d'un accomplissement de soi, la joie d'une épreuve qui, quoique douloureuse, demeure l'axe d'une orientation prédicative qui s'impose au récit second sur l'excision. C'est l'objet d'étude de la première partie de cet article.

Incirconcis, Waiyaki se fait ombre dans un espace où son existence se mesure aux épisodes laborieux de tâches quotidiennes. Sa vie s'écoule entre l'ennui de la routine et l'amertume d'un interdit d'accès à l'univers des hommes de la société Kikuyu. Il vit dans une angoisse existentielle et s'impatiente de se voir autoriser à couper le cordon ombilical qui le lie à sa « vie de boy » et à son « innocence ». Et c'est l'intensité de cette 
impatience qui sera à la mesure de la grandeur de sa joie une fois initié. C'est ce que nous allons analyser dans le second point de notre contribution.

\section{De quelques notions théoriques}

Nous recourrons aux moyens d'analyse des théories sémiotiques pour traiter de la joie christique et coutumière dans le deuxième roman de Ngugi en essayant de comprendre comment et pourquoi ces personnages éprouvent les sentiments antagoniques d'une douleur mortelle et d'une joie ataraxique, comment la joie peut s'exprimer en la forme accomplie d'une liberté à la fois "laïque » et coutumière. En effet, comme l'explique Jacques Fontanille, dans son manuel Sémiotique du discours (1999) :

La sémiotique est devenue une sémiotique du discours en redonnant toute sa place à l'acte d'énonciation, aux opérations énonciatives, et pas seulement à la représentation du "personnel" d'énonciation (narrateurs, observateurs, etc.) dans le texte: elle est alors à même d'aborder le discours littéraire non seulement comme un énoncé qui présenterait des formes spécifiques, mais aussi comme une énonciation particulière, une " parole littéraire ", comme dirait Jacques Geninasca. ${ }^{2}$

Il est pertinent de souligner que cette sémiotique ne se veut pas une nouvelle théorie sémiotique, comme le précise J. Fontanille dans l'introduction à son ouvrage (1999) : «Il ne s'agit donc pas de proposer une théorie sémiotique du discours littéraire de plus (...) mais de montrer quel peut être l'apport, en termes de méthode, d'un point de vue sémiotique sur la question posée » (idem).

Ce « point de vue », poursuit-il, est celui d'un « discours en acte ", « discours vivant, de la signification en devenir» dont le style est ainsi défini, dans cette même introduction : «le style (Le style, l'identité et les formes de vie) sera traité comme une identité en construction, pour une instance de discours en devenir dans les actes d'énonciation "; d'où l'importance du sujet de la " parole littéraire ", c'est-à-dire ici, du personnage (Muthoni - Waiyaki).

Dans notre cas, l'étude se focalisera sur les trois axes sémiotiques, complémentaires, de la "présence», de la "visée intentionnelle» et de la "saisie». et de la "visée intentionnelle » de la joie.

La présence, toujours selon Jacques Fontanille (2003 : 38) réside dans le fait de

Percevoir quelque chose avant même de le reconnaitre comme une figure appartenant à l'une des deux macro-sémiotiques, c'est percevoir plus ou moins intensément une présence. (...) [Cette présence est] quelque chose qui, d'une part occupe une certaine position, relative à notre propre position, et une certaine étendue, et qui, d'autre part, nous affecte avec une certaine intensité (...). La présence, qualité sensible par excellence, est donc une première articulation sémiotique de la perception.

Cette présence, ou plutôt ce sentiment de présence ou pressentiment déclenche une seconde articulation sémiotique de la perception, la « visée intentionnelle » : « L'affect qui nous touche, cette intensité qui caractérise notre relation avec le monde, cette tension en direction du monde est l'affaire de la visée intentionnelle » (Fontanille, $2003: 39)^{3}$.

La troisième articulation est la « saisie » à travers laquelle s'appréhendent « la position, l'étendue et la quantité [qui] caractérisent en revanche les limites et les propriétés du domaine de pertinence, c'est-à-dire celles de la saisie » (Fontanille, $2003: 39$ ).

La visée (intensité variable de l'affect) et la saisie (l'entendue de l'affect) forment

Un système de valeurs [qui] résulte donc de la conjugaison d'une visée et d'une saisie, une visée qui guide l'attention sur une variation, dite intensive, et une saisie qui met en relation cette première variation avec une autre, dite extensive. (Fontanille, $2003: 40$ ) 
Pour une exploitation pertinente de ces notions, nous prenons appui sur la conception sémiotique du récit en nous référant, notamment, à la méthodologie d'analyse proposée par Nicole Everaert-Desmedt dans son ouvrage Sémiotique du discours (2004). Elle définit le récit ainsi :

Nous définissons le récit comme la représentation d'un événement. (...) Précisons: un événement est une transformation, un passage d'un état $S$ à un état $S$ ' (...). [Un événement devient récit lorsqu'il est] représenté, rapporté par quelqu'un (raconté, filmé, mis en scène,...). (...) Toute représentation est déjà interprétation. (2004:13)

Cette définition globale est exploitée à trois niveaux - narratif, figuratif et thématique qui «entretiennent des rapports entre eux pour constituer le "parcours génératif de la signification" » (Fontanille, 1999 : 14), et qui « permettent de décrire (...) l'engendrement de la signification dans un texte » (idem.).

Certes, ces niveaux entretiennent des rapports d'interdépendance, mais pour des nécessités méthodologiques, nous allons réserver un point au parcours figuratif parce que les composantes sémiotiques de l'espace jouent un rôle primordial dans l'expression de la joie transformationnelle dans le corpus que nous avons choisi.

\section{Valeur sémiotique de « la belle souffrance »}

\section{II.1. Présence, visée et saisie d'un vouloir ineffable}

3 Le dessein de se faire exciser envahit la fille du pasteur et l'obnubile. Elle mûrit son idée en silence. Le désir de devenir une femme aux yeux de la tradition de Kameno devient présence en tant que "première articulation sémiotique de la perception». Cette présence se mue donc en une «qualité sensible par excellence ». Une intensité d'affects lie Muthoni à son rêve. Elle brise le silence et s'en ouvre à sa grande sœur Nyambura :

I have to tell you something. (...) I thought and thought again about it. I have not been able to eat or sleep properly. My thoughts terrify me. But I think now I have come to a decision.

(...) Nyambura, I want to be circumcised. (RB : 24-25) ${ }^{4}$

Muthoni perçoit ici l'intensité de la présence qu'elle met en relation avec la variante croyance dans une visée intentionnelle ("I want to be circumcised» - je veux être excisée). En effet, la visée qui implique la figure de l'interprétant ${ }^{5}$ mesure l'intensité par laquelle une relation entre le sujet Muthoni, son désir, et son espace est établie. Le bornage de cette étendue fait appel à une saisie caractérisant le fondement et l'étendue du projet du sujet de faire.

L'espace de la présence du désir de Muthoni devient intelligible ${ }^{6}$, et des transformations majeures se profilent à l'horizon.

Muthoni est face à une catégorisation axiale complexe avec, d'une part, un agrégat d'affects autour de la notion sacrée des traditions et, d'autre part, un élan ecclésiastique qui déprécie toute pratique traditionnelle jusqu'à l'interdire formellement. Sa sœur, Nyambura le lui fait comprendre :

You are a Christian. You and I are now wise in the way of the white people. Father has been teaching us what he learnt at Siriana. and you know the missionaries do not like the circumcision of girls. Father has been saying so. Besides, Jesus told us it was wrong and sinful. $(\mathrm{RB}: 25)^{7}$

Dans l'optique de la sœur, deux systèmes de valeurs s'affrontent dans un face-à-face intense de ce qui se donne comme le «bien» contre le «mal». Par conséquent, le devenir de Muthoni s'évalue en terme de choix et de responsabilité appréhendés en termes de saisie et d'étendue, de faire-être et de ne pas-faire-être. La fille du pasteur est 
plongée dans un dilemme où les termes d'opposition lui ravissent toute marge de liberté dans un espace catégorielle de valeurs. Elle se laisse alors emporter par l'intensité d'un rêve et l'étendue d'une sanction finale. Elle trouve sa voie en s'affranchissant de l'opposition privative de sa famille et en imposant la saisie de son vouloir. Elle s'en va à Kameno (le village de la tribu) pour harmoniser les contraires : la coutume et les interdits d'une religion chrétienne. Le signe de la réconciliation sera sa croix de martyr qu'elle portera avec joie et optimisme. D'où la corrélation entre la joie d'un devoir à accomplir et le courage d'une décision à prendre. Ce syncrétisme repose, pour le sujet, sur la cohésion des deux croyances dont Muthoni sollicite la reconnaissance sociale: "Father and mother are circumcised. Are they not Christians? Circumcision did not prevent them from being Christians " $(\mathrm{RB}, 26)^{8}$. Mais une rupture se produit d'où se dégage l'expression d'un accomplissement de soi, la joie d'une épreuve qui, quoique douloureuse, demeure l'axe d'une orientation prédicative qui s'impose au récit. Elle s'exprime comme le point nodal d'un champ positionnel autour duquel gravitent l'intensité et l'étendue émotionnelle de la fille du pasteur. Celle-ci entame un mouvement létal de conjonction et de disjonction dans un état d'esprit caractérisé par la joie de souffrir et de mourir au nom d'une cause qu'elle croit noble.

\section{II.2. Le méta-sujet et son faire transformateur à l'épreuve d'une douleur-joie mortifère}

L'analyse des actes de langage de Muthoni révèle qu'elle est l'expression d'un espace où se produisent des "événements syntaxiques " ${ }^{9}$. Elle est à la fois un actant-sujet et un actant-objet : "I want to be a woman, I - I want to be a woman. I want to be a real girl, a real woman, knowing all the ways of the hills and ridges " $(\mathrm{RB}: 26)^{10}$.

Son énoncé prend la valeur d'un acte de langage réfléchi qui se manifeste dans la joie d'un faire-être, faisant ainsi de la fille du pasteur « un lieu-dit acteur syncrétique » ${ }^{11}$. La douleur de l'excision prend alors valeur d'importance dans sa dimension fonctionnelle, déterminant chez elle un type de comportement joyeux.

La structure actantielle du récit second se trouve bouleversée par la structure actorielle qui donne forme à l'«organisation réflexive de l'univers individuel ${\aleph^{12}}$ de la fille excisée. L'univers culturel de Kameno se lit dans une certaine mesure dans la narrativisation psycho-sémiotique du monde du dedans de Muthoni. Au lieu de se soustraire aux tourments et de s'éloigner de l'espace des traditions qui la détermine à souffrir, Muthoni s'exprime dans la volonté de soustraire son corps au "péché ». Son bonheur, sa joie se déroulent hors du temps et hors du lieu caractéristiques d'un monde de douleurs; un monde que son père, le révérend, voit au prisme de l'Egypte pharaonique : «Let her (Muthoni) go back to Egypt. Yes. Let her go back. He (Joshua) would travel, on to the new Jerusalem » (RB : 36) ${ }^{13}$. Excisée, Muthoni réconcilie le Dieu Murugu et celui de Jésus Christ pour faire naître, à travers sa personne, un interprétant (son statut de fille excisée) qui fait appel à un signe argumental symbolique pour dire une nouvelle signification : être chrétienne et excisée signifie incarner la beauté de sa tribu dans cette société Kikuyu. De par cet acte, la fille connaît des transformations et fait jouer à son énoncé une fonction de conjonction et de disjonction (Greimas, 1973) ${ }^{14}$. Ce qui confère à Muthoni le statut d'un méta-sujet opérateur dont l'action peut être exprimée en une équation sémiotique type explicitée par Greimas (Greimas, 1973) ${ }^{15}$ : $\mathrm{F}$ (transformation) $\left(\mathrm{S}_{1} \cup \mathrm{OV}_{1}\right) \longrightarrow\left(\mathrm{S}_{1} \cap \mathrm{OV}_{2}\right)^{16}$. Le méta-sujet agit à travers un faire transformateur $\left(\mathrm{S}_{1} \cup \mathrm{OV}_{1}\right)$, entraînant une transformation transitive ${ }^{17}\left(\mathrm{~S}_{1} \cap 0 \mathrm{~V}_{2}\right)$ 
exprimée dans cet énoncé, «I'm a Christian ; a Christian in the tribe » (RB, 53), qui est une conséquence de la transformation (F).

L'épreuve de l'excision s'exprime alors comme une narrativité (Greimas, 1970:28) et une réalisation au sens de Greimas, à savoir "une transformation qui établit la conjonction entre le sujet et l'objet: Real =F trans $\left[\mathrm{S}_{1} \longrightarrow \mathrm{O}_{1}(\mathrm{~S} \cup \mathrm{O})\right]$ » (Greimas, $1973: 20$ ).

La beauté de sa tribu se lexicalise et s'identifie comme une "valeur réalisée » définie comme « la valeur investie dans l'objet au moment (= dans la position syntaxique) où celui-ci se trouve en conjonction avec le sujet » (Greimas, $1973: 20$ ).

Cette conjonction est signifiée par la joie exprimée d'autant plus que la disjonction de Muthoni de cette partie de son corps ne signifie guère le reniement d'une conjonction avec le Christ.

Greimas précise dans ce sens que : « la disjonction étant la dénégation de la conjonction n'est pas l'abolition de toute relation entre les deux actants : autrement, la perte de toute relation entre sujets et objets aboutirait à l'abolition de l'existence sémiotique » " (Greimas, $1973: 28-29$ ).

Muthoni reste en relation avec la religion du Christ, faisant ainsi des croyances des étants sémiotiques qui opèrent des modes d'expressions différents dans leur articulation conjonctive illustrée dans cet énoncé : "I'm a woman and I will grow big and healthy in the tribe. (...) . Tell Nyambura I see Jesus and I am a woman, beautiful in the tribe » (RB : 53) ${ }^{18}$. Cet attachement à ses croyances chrétiennes virtualise sa disjonction avec l'église et fait de son énoncé un déclaratif d'état qui fusionne virtualisation et réalisation. Cela fait du sujet Muthoni un "mode de manifestation" (Greimas, 1973: 24) de l'excision sur un axe sémantique investi d'une valeur qu'est la joie dans la douleur sacrificielle; propos synthétisé dans le tableau suivant:

\begin{tabular}{|c|c|c|}
\hline EPREUVES & ACQUISITIONS & PRIVATIONS \\
\hline EXCISION & $\begin{array}{l}\text { SYNCHRÉTISME DES DEUX RELIGIONS: } \\
\text { «I SEE JESUS AND I AM A WOMAN, } \\
\text { BEAUTIFUL IN THE TRIBE» }\end{array}$ & $\begin{array}{l}\text { UNE PARTIE SOUILLÉE DE SON } \\
\text { CORPS }\end{array}$ \\
\hline $\begin{array}{lll}\text { EXCOMMUNION } & \text { DE } & \text { LA } \\
\text { COMMUNAUTÉ } & & \\
\text { CHRÉTIENNE } & & \end{array}$ & $\begin{array}{ll}\text { DEVIENT } & \text { UNE } \\
\text { TRADITIONNELLE } & \text { FEMME }\end{array}$ & $\begin{array}{lrr}\text { RENONCIATION } \quad \text { A } & \text { LA } \\
\text { RELIGION CHRÉTIENNE } & \text { QUI } \\
\text { INTERDIT L'EXCISION } & \end{array}$ \\
\hline $\begin{array}{l}\text { EXCLUSION DE LA } \\
\text { COMMUNAUTÉ } \\
\text { TRADITIONNELLE }\end{array}$ & ELLE EST TOUJOURS CHRÉTIENNE & $\begin{array}{l}\text { RENONCIATION À L'INTERDIT } \\
\text { COUTUMIER QUI } \\
\text { EXCLUT LES FILLES } \\
\text { CHRÉTIENNES DE LA } \\
\text { PRATIQUE DE L'EXCISION }\end{array}$ \\
\hline
\end{tabular}

Ces transformations du point de vue syntagmatique s'opèrent non seulement sous un angle d'acquisition, mais également de « «manque propien» (Greimas, 1970: 40) qui reflète un aspect positif à travers lequel est articulé le vouloir du sujet Muthoni.

Le renoncement, au contraire du son statu quo ante, a conduit la fille à s'attribuer la valeur joie malgré la condamnation dont elle fait l'objet dans les deux camps. Elle devient alors un acteur inclusif des sèmes /femme/, /joie/, /chrétienne/, / 
traditionnaliste/. Ces sèmes entretiennent, au plan syntaxique, une relation hyponymique (indépendante), mais aussi antinomique (relation de conjonction et de disjonction) au niveau sémantique. La fille du pasteur se retrouve au centre d'une occurrence d'inclusion où la joie christique fait figure d'actant qui s'interprète au niveau sémantique comme un sème, un objet de valeur positif. D'où l'articulation sémiotique suivante :

$\mathrm{S}_{1}$ (Muthoni) $\cup \mathrm{OV}^{+} 1$ (joie dans la douleur et jusqu'à la mort).

\section{Espace figuratif d'une renaissance}

Nous envisageons d'étudier ce niveau du récit en nous référant au parcours d'un personnage masculin, Waiyaki, en rapport avec le rituel de la circoncision.

\section{III.1. Espace du dedans : niveau génératif de la joie transformationnelle}

6 L'icono-texte relatif à l'axe thématique de la joie coutumière revêt une dimension narrative et figurative dont l'analyse met en surface des disjonctions actorielles, spatiales et temporelles.

L'épreuve de la circoncision, à la fois physique et douloureuse, en appelle à la participation d'acteurs et d'actants en interactions syntagmatique et paradigmatique dans des espaces différents : l'espace du dedans, qui a pour correspondant perceptible la maison, fait face à l'espace du dehors dont le représentamen se lit sous le signe du fleuve.

7 Dans l'espace du dedans les indications spatiales s'articulent en :

A. patience $v s$ impatience

B. Innocence vs initiation

8 Une telle articulation se lit dans des expressions telles que : "Days came and went; and still it was the same life " $(\mathrm{RB}, 13)^{19}$, "Waiyaki joined again in the daily rhythm of life » (RB : $13)^{20}$.

L'espace du dedans réfère à une relation de conjonction: Waiyaki fait corps avec les objets de valeur impatience et innocence, tandis que l'espace du dehors renvoie au rêve de Waiyaki de se mettre en jonction avec l'objet de valeur initiation.

Ainsi, l'opposition figurative innocence vs initiation qui détermine un épisode où le mouvement fait appel à un sentiment de joie immense chez le non-initié.

$\mathrm{Mu}$ par son désir d'accéder au statut d' "homme » dans sa tribu, Waiyaki s'empresse d'accueillir et de se réjouir de toute idée le rapprochant de l'événement : «Some a glow of pride was beginning. He was ready for initiation $"(\mathrm{RB}: 12)^{21}$.

$\mathrm{Au}$ niveau syntagmatique les éléments du dedans s'agencent pour ainsi tracer un parcours configuratif où se lisent les motifs de " tutelle " et d'« impatience». Ces motifs impliquent des données figuratives que le narrateur emploie pour produire un « effet de réel ».

En effet, la présence de Waiyaki dans l'espace du dedans est signe d'un statut social : le jeune garçon n'est pas un homme au sens coutumier des Kikuyu. Il est placé sous la responsabilité de ses parents et demeure éloigné des secrets de la vie, de la culture et 
autres connaissances socio-culturelles de son ethnie. Son innocence le prive du pouvoir d'acquisition de compétence et de performance.

D'où l'impatience du jeune garçon à se libérer de son statut d'incirconcis pour une renaissance, comme il le déclare à sa mère : «I must be born again » $(R B: 11)^{22}$. Son statut est un fait qui s'oppose alors à son vouloir et donc à sa joie. Le dedans l'enferme avec son désir et fait de son sentiment d'impatience et d'ennui une composante fonctionnelle de l'espace "maison ». Waiyaki rêve d'un ailleurs où il pourra connaître une réalisation de soi, une renaissance par laquelle il se mettra, avec joie, en jonction avec le statut d'homme, initié, dans sa communauté.

\section{III.2. Espace du dehors : référent médiat d'une douleur-joie coutumière}

10 Ngugi, dans $R B$, utilise l'image du fleuve comme un moyen euristique afin de transmettre un message cultuel.

Pour décoder les aspects sémiotiques des éléments fluviaux, dans The River Between, nous allons adopter une démarche analytique qui fait appel à la charge sémantique de " l'icône métaphorique » (Kibedi et al, $1981: 250$ ). Selon A. Kibédi Vergas et al. : « Ce qui caractérise ce genre d'icônes, c'est que la relation de ressemblance ne s'établit pas entre le signe et son référent, mais entre deux référents qui sont tous les deux dénotés par le même signe" (idem.).

Peirce, quant à lui, décrit ce type d'icônes comme des métaphores, c'est-à-dire ce sont «celles qui représentent le caractère représentatif d'un représentamen [ou signe] en représentant un parallélisme dans quelque chose d'autre ${ }^{23}$.

L'espace du dehors limite la narration du récit global et focalise les acteurs dans un parcours de soustraction et d'acquisition de valeurs. La narration d'un événement, dont l'expression et le contenu concourent à peindre un acteur heureux dans l'expérience d'une douleur physique, se construit dans une articulation sémiotique d'interactions verbale et physique, lesquelles impliquent des macro-transformations chez les acteurs qui les intègrent dans un processus de conjonction ou de disjonction.

Dans l'espace du fleuve, sujet de réalité socio-culturelle, s'accomplit un vouloir coutumier. De ce fait, son iconicité porte la marque d'un référent médiat en une pratique culturelle. Avant de subir l'ablation tant rêvée, Waiyaki est amené au fleuve Honia, symbole de pureté et de sacralité, pour prendre un bain purificateur. Le jour fatidique, sa joie est immense. Le bonheur de ce rêve à accomplir lui est décrit en termes sécurisants: "Waiyaki whose head had had been shorn of hair trailed behind her (mother) as a little child would follow his mother $\gg(\mathrm{RB}: 12)^{24}$.

Il fait mouvement vers l'espace du dehors où il doit recevoir le signe de la circoncision comme sceau de son titre d'homme libre et responsable. D'où sa joie de marcher sur le tracé de la foi coutumière, à l'instar de Muthoni. Ce mouvement vers une mort symbolique prend une forme narrative d'une iconicité structurale, et le fleuve Honia signifie un espace investi de valeurs traditionnelles de l'essence de l'être dans la société Kikuyu. Ses eaux iconisent alors la renaissance. Il est le signe d'un espace d'adieu, une frontière-contact entre une vie anté et une existence posté. De ce fait, il est associé aux aspects temporels et existentiels du bonheur de renaître du personnage Waiyaki : «Old Waiyaki is born. Born again to carry on the ancient fire $"(\mathrm{RB}: 12)^{25}$. 
L'espace fluvial est porteur de la joie d'être ; c'est un espace de félicité dont les valeurs lient le Kikuyu au sens profond de l'identité coutumière. Après le bain purificateur, Waiyaki sort du fleuve, débarrassé de la souillure de son état d'incirconcis, pour subir l'épreuve de la circoncision: se faire couper le prépuce, symbole de son impureté. Il vise la victoire dans l'épreuve pour faire honneur à sa personne et à sa famille :

Waiyaki had waited for this day, for this very opportunity to reveal his courage like a man. This had been the secret ambition of his youth. (...) now time had come (...). He just stared into space, fear giving him courage. His eyes never moved. (...). The knife produced a thin sharp pain as it cut through the flesh. (...). Blood trickled freely on the ground (...) Around him women were shouting and praising him. The son of Chege had proved himself. Such praises were lavished only on the brave. $(\mathrm{RB}: 45)^{26}$

Son vouloir se réalise et fait de lui un homme accompli. Après s'être éprouvé dans l'attente, le voilà qui reçoit la couronne de la vie et prépare sa voie dans la vie active.

Affranchi, dans cet espace du dehors, Waiyaki goûte au fruit de la connaissance et à la vie d'homme libre et heureux. La joie saisissant l'occasion d'une expression coutumière séduit Waiyaki par le commandement de son essence qui le fait mourir dans le sable boueux de Honia River afin de le faire revivre dans sa communauté kikuya. De plus, il est doté du pouvoir d'exprimer ce qu'il est devenu et ce qu'il représente désormais dans sa société. Honia se mue en un pont de douceur qui relie la mort à la vie. Le désir de mort engendre la joie de vivre chez le fils de Chege; cela fait du signe du fleuve l'expression d'une spiritualité et d'une humanité. A travers le fonctionnement iconique et métaphorique du fleuve, l'espace du dehors se solidarise du changement de statut de Waiyaki en établissant un lien avec son espace et l'initié.

Ainsi, le thème de la joie s'est exprimé avec étendue et intensité dans The River Between où les coutumes africaines sont situées à la croisée des chemins de civilisations différentes. Par l'excision, la fille du pasteur vise à connaître un accomplissement. Cette épreuve est insigne de son supplice qu'elle savoure avec bravoure et joie. Elle est rayonnante dans son sacrifice héroïque. Ce sacrifice la met en jonction avec la valeur positive des traditions des Kikuyu, lesquelles, à leur tour, sont mises en relation jonctive avec les valeurs chrétiennes. Muthoni, grâce à ses deux croyances, qui l'amènent à Kameno et à Makuyu, subit le destin d'une mort rédemptrice. Sa mort délivre un message de joie. Elle annonce la bonne nouvelle: elle a réussi à mettre sa foi chrétienne et sa foi paganiste en accord dans un syncrétisme salutaire, purifié de tout extrémisme. Elle a connu la mort, certes, mais pour entrer en jonction avec la valeur de la liberté et du salut ; d'où la joie d'une mission accomplie, d'un objectif atteint.

Contrairement à Muthoni, qui s'est efforcée de trouver le point de jonction de deux religions qui s'entrecroisent, Waiyaki, lui, a choisi la tradition de ses ancêtres pour se réaliser dans la joie d'une épreuve qui le porte à un haut niveau social. Sa circoncision lui a ouvert les perspectives d'un renouveau, d'une autre vie.

Son séjour dans un espace fluvial et la disjonction d'avec son prépuce n'ont d'autre but que de le faire passer d'une mort symbolique à une vie nouvelle. Cette purification de toute sa souillure physique et morale de sa vie de profane, est ce qui explique la joie de s'accomplir dans les secrets de sa coutume. Sa joie s'oppose à celle de Muthoni par sa visée. Alors que Muthoni se réjouit d'une mort physique certaine, conséquence de son projet christique, Waiyaki se réjouit d'une mort symbolique pour une vie spirituelle ancrée dans la racine identitaire de la tradition de sa tribu. L'expression de ces deux façons de mourir et de connaître la joie s'inscrit dans la difficile question des relations interculturelles entre le Sud et le Nord, entre le marteau de l'ouverture et l'enclume de l'enracinement. Nous avons tenté de le démontrer à travers l'étude de la particularité 
sémiotique de la narrativité de The River Between que nous définirons comme une " forme mixte, à la fois psycho- et socio-sémiotique (correspondant à l'ensemble des pratiques inter-individuelles)» (Greimas, $1973: 19)$.

\section{BIBLIOGRAPHIE}

EVERAERT-DESMEDT N., Sémiotique du récit, Paris, De boeck, $3^{\mathrm{e}}$ édition, 2004.

FARAH Nuruddin, Sardines, London, Allison and Busby, 1981. FONTANILLE Jacques, Sémiotique et littérature : essai de méthode, P.U.F., 1999.

FONTANILLE Jacques, Sémiotiques du discours, Paris, Presses Universitaires de Limoges, 2003.

GREIMAS, A. J., Sens II : essais sémiotiques, Paris, Seuil, 1970. GREIMAS, A. J., « Un problème de sémiotique narrative : les objets de valeur », in Langages, $\mathrm{N}^{\circ} 31$, volume 8, 1973, pp. 13-35.

HENAULT Anne, Narratologie, sémiotique générale : les enjeux de la sémiotique, Paris : PUF, 1983.

KERBRAT-ORECCHIONI Catherine, L'Énonciation. De la subjectivité dans la langue, Paris, Nathan, 1985.

KIBEDI VERGA A. et al., Théorie de la littérature, Picard, 1981.

THIAM Awa, La Parole aux négresses, Denoël, 1978.

THIBAUD P., « La notion peircienne de métaphore », in Histoire Épistémologie Langage, Tome 16, fascicule 1, 1994. pp. 123-136.

CORPUS : NGUGI Wa Thiong'o, The River between, London, Heinemann, 1965, 1989.

\section{NOTES}

1. Nous traduisons : « mais père ne le permettra pas »

2. L'introduction du manuel est disponible sur le site: < https://www.unilim.fr/pages_perso/ jacques.fontanille/textes-pdf/BSemiotiquediscoursintro.pdf >

3. Fontanille cite l'exemple du narrateur d'A la Recherche du temps perdu de M. Proust qui, pendant l' "épisode de la madeleine", "a ressenti l'effet de la madeleine, il a apprécié l profondeur où le souvenir est enfoui; en reconnaissant l'effet gustatif comme associé à une saveur, il a du même coup attribué une valeur intentionnelle à cette présence sensible » (2003:108).

4. Nous traduisons : «Je dois vous dire quelque chose. (...) J'y ai pensé et y pense de nouveau. Je n'ai ni pu manger ni dormir correctement. Mes pensées me terrifient. Mais je pense maintenant que j'en suis venue à une décision. (...) Nyambura, je veux être excisée »

5. «En termes peirciens, la visée rappelons-le, caractériserait l'interprétant, et la saisie, le fondement » (Fontanille, $2003: 39$ ).

6. « (...) Un système de valeurs ne peut prendre corps que si des différences apparaissent et si ces différences forment un réseau cohérent : c'est la condition de l'intelligible » (Fontanille, 2003 : 39). 
7. Nous traduisons : «Vous êtes chrétienne. Toi et moi sommes maintenant sages comme les Blancs. Le père nous a enseigné ce qu'il a appris à Siriana. Et tu sais que les missionnaires n'admettent pas la circoncision (l'excision) des filles. Le père l'a dit. De plus, Jésus nous dit que c'est un péché. »

8. Nous traduisons : «Père et mère sont circoncis. Ne sont-ils pas chrétiens ? La circoncision ne les a pas empêchés d'être chrétiens"

9. Cette terminologie est empruntée à A.J. Greimas : " "Jean se torture tout le temps". L'analyse superficielle de cet énoncé linguistique nous révèle qu'à l'intérieur d'un acteur dénommé Jean et considéré comme un lieu où se produisent des événements syntaxiques, Jean en sa qualité d'actant sujet, torture le même Jean pris comme l'actant objet » (Greimas, 1973 : 18).

10. Nous traduisons : «Je veux être une femme, je - je veux être une femme. Je veux être une fille authentique, une femme authentique, connaissant tous les chemins des collines et des crêtes »

11. On voit que le statut de ce que l'on appelle un énoncé réfléchi s'interprète aisément par l'inscription d'un énoncé syntaxique quelconque dans le lieu-dit acteur syncrétique (...) » (Greimas, $1973: 18$ )

12. «(...) On voit que c'est le type de rapports entretenus entre la structure actancielle et la structure actorielle qui détermine, comme des cas-limites, tantôt l'organisation réflexive des univers individuels tantôt l'organisation transitive des univers culturels, et qu'une même syntaxe est susceptible de rendre compte de la narrativisation psyco-sémiotique ("la vie intérieure") et de la narrativisation socio-sémiotique (mythologies et idéologies) (...)» (Greimas, $1973: 19$ ).

13. Nous traduisons : «Laissez-la (Muthoni) retourner en Égypte. Oui. Laissez-la y retourner. Il (Joshua) ira de nouveau à Jérusalem »

14. «On comprendra notre définition provisoire de la narrativité qui consiste en une ou plusieurs transformations dont les résultats sont des jonctions, c'est-à-dire soit des conjonctions, soit des disjonctions des sujets d'avec les objets » (Greimas, $1973: 20$ ).

15. «Enoncé de faire de type: $F$ transformation ( $\mathrm{S} 1 \mathrm{O} 1)$ où $\mathrm{S} 1$ est le sujet opérant la transformation et 01 est l'énoncé d'état auquel aboutit la transformation » (Greimas, $1973: 20$ ).

16. Nos équations sémiotiques sont, certes, inspirées de celles de Greimas mais plus précisément de celles de A. Hénault (1979 et 1983) : S1 = sujet (le sujet peut être un sujet de faire ou un sujet d'état), $\mathrm{S} \cup \mathrm{O}=$ énoncé d'état disjonctif, $\mathrm{S} \cap \mathrm{O}=$ énoncé d'état conjonctif, $\mathrm{O}=$ objet et $\mathrm{V}=$ valeur.

17. Selon Greimas $1970: 37$, il s'agit, sur le plan figuratif, d'une transformation sous-tendue par une attribution d'une valeur à un objet.

18. Nous traduisons: «Je suis une femme et je serai grande et saine dans la tribu. (...). Dites à Nyambura que j'ai vu Jésus et que je suis une femme, belle au sein de la tribu »

19. Nous traduisons : «Les jours passaient et c'était toujours la routine »

20. Nous traduisons : «Waiyaki retouve de nouveau le rythme quotidien de vie »

21. Nous traduisons: «Il commençait à ressentir un sentiment de fierté. Il était prêt pour l'initiation "

22. Nous traduisons : " Je dois naître à nouveau »

23. Pearce classe les icônes en trois catégories : «On peut en gros diviser les hypoicônes suivant le mode de priméité auquel elles participent. Celles qui font partie des simples qualités, ou priméités premières, sont des images; celles qui représentent les relations, principalement dyadiques, ou considérées comme telles, des parties d'une chose par des relations analogues dans leurs propres parties sont des diagrammes; celles qui représentent le caractère représentatif d'un représentamen en représentant un parallélisme dans quelque chose d'autre sont des métaphores (1902 : 2.277). Cité par P. Thibaud (1994:126).

24. Nous traduisons : "Waiyaki dont les cheveux ont été rasés traînait derrière elle (la mère) comme un petit enfant suivrait sa mère "

25. Nous traduisons: «Le vieux Waiyaki est né. Né de nouveau pour porter le flambeau de la tradition» 
26. Nous traduisons : «Waiyaki avait attendu ce jour, cette occasion, pour montrer son courage comme un homme. C'était son ambition secrète depuis qu'il était (tout) jeune..(...) Le temps était venu (...). Il a juste regardé fixement dans l'espace, la crainte lui donnant du courage. Ses yeux n'ont jamais cillé. (...). Le couteau a produit une douleur vive quand il a coupé la chair.(...). Le sang a coulé goutte à goutte librement sur le sol (...). Autour de lui, des femmes criaient et louaient ses qualités. Le fils de Chege avait fait ses preuves. Ces louanges n'ont fait que se répandre sur un valeureux »

\section{RÉSUMÉS}

Omniprésent dans les ouvrages de Ngugi Wa Thiong'o, le thème de la joie demeure un signe pertinent dans la chaîne des interactions des personnages de The River Between $(1965,1989)$. Notre objectif dans cet article est de mettre en évidence l'aspect sémiotique de la narration de la joie « christique » et « coutumière ». Nous soulignerons également la distinction entre les différentes formes de joie évoquées et leurs portées sociales et religieuses au sein du peuple Kikuyu du Kenya. Pour ce faire, une étude de la thématique de l'excision et de la circoncision sous-tendra le point nodal de notre analyse.

Omnipresent in Ngugi Wa Thion'go's fictional work, the theme of joy stands as a meaning ful sign in the verbal and physical interactions of the characters of his novel The River Between. Our objective in this paper is to highlight the semiotic aspect of the narration of "Chrislike" and "customary" joys. We will also point out the difference between the already mentioned forms of joy and their social and religious impact on the Kikuyu society. To achieve this objective, a study of the social practices of female genital mutilation and circumcision will underlie our analysis.

\section{INDEX}

Keywords : semiotics, style, presence, seizure, intention, The River between, Ngugi (Wa Thiong'o)

Mots-clés : sémiotique, style, présence, saisie, visée, The River beetween, Ngugi (Wa Thiong'o)

\section{AUTEUR}

\section{ABIB SENE}

Université Gaston Berger Saint-Louis - Sénégal 